\title{
The next three years
}

$\mathrm{I}$ $t$ is a great honour to be elected by the committee as Chairman of the GPIAG. Before looking at the direction of the group over the next three years it is appropriate to consider what has already been achieved and to pay tribute to my predecessor, Dermot Ryan. During his chairmanship, Dr Ryan was instrumental in the development of the GPIAG Chair in Primary Care Respiratory Medicine, he raised the profile and membership of the group and developed key relationships with other professional respiratory bodies. The other longstanding strengths of the group are our journal and research unit in Dundee. I believe that a number of further developments in our core strengths are desirable; the biggest of which is our membership and I would like to outline some of the ways that we aim to develop this.

With the advent of the National Institute of Clinical Excellence (NICE) and following our successful involvement with other professional groups we aim to elect GPIAG representatives onto other professional bodies. I have already had significant expressions of interest for this voluntary work from members of the group, but welcome more.

As part of the GPIAG's commitment to education we are planning a Primary Care International Respiratory Conference this year which will go beyond the remit of our annual scientific meeting. The meeting, to be held in the UK, will include a variety of primary care forums with international primary care speakers and guests.

An initiative that we are piloting is the evaluation of a Regional Practice-based Respiratory Education Programme which, if successful, will be taken nationwide.
Much of our development over the last 12 years owes a great debt to our largest sponsors, Allen and Hanburys. In the light of changing times in medicine and our need to be seen as independent we have already sourced much funding from other pharmaceutical companies especially in the consortium funding of the GPIAG Chair in Primary Care Respiratory Medicine. However, to pursue future planned activities of research and education the group will need to generate further funding and the committee are actively developing a business plan to secure multifunding from a number of organisations. We are also considering and will take the membership's views on a membership fee.

The GPIAG and in particular the Research Unit in Dundee has led the way in information technology with our website, availability of our journal on-line and question and answer forum. We are planning to develop this further and evaluate the prospect of a fully interactive on-line journal.

I am proud to say that over the years the GPIAG and its membership has led the way with much excellent research relevant to general practice. This is fundamental to our future as a group and I believe that the GPIAG Primary Care Respiratory Chair at Aberdeen will enable us to take this agenda forward in conjunction with our research unit at Dundee and our recently formed research network.

I would welcome comments from the membership and others interested in our activities via our secretariat MMI on 01225858880 (triciabryant@msn.com).

David Price

GPIAG Chairman

dprice@gpiag-asthma.org

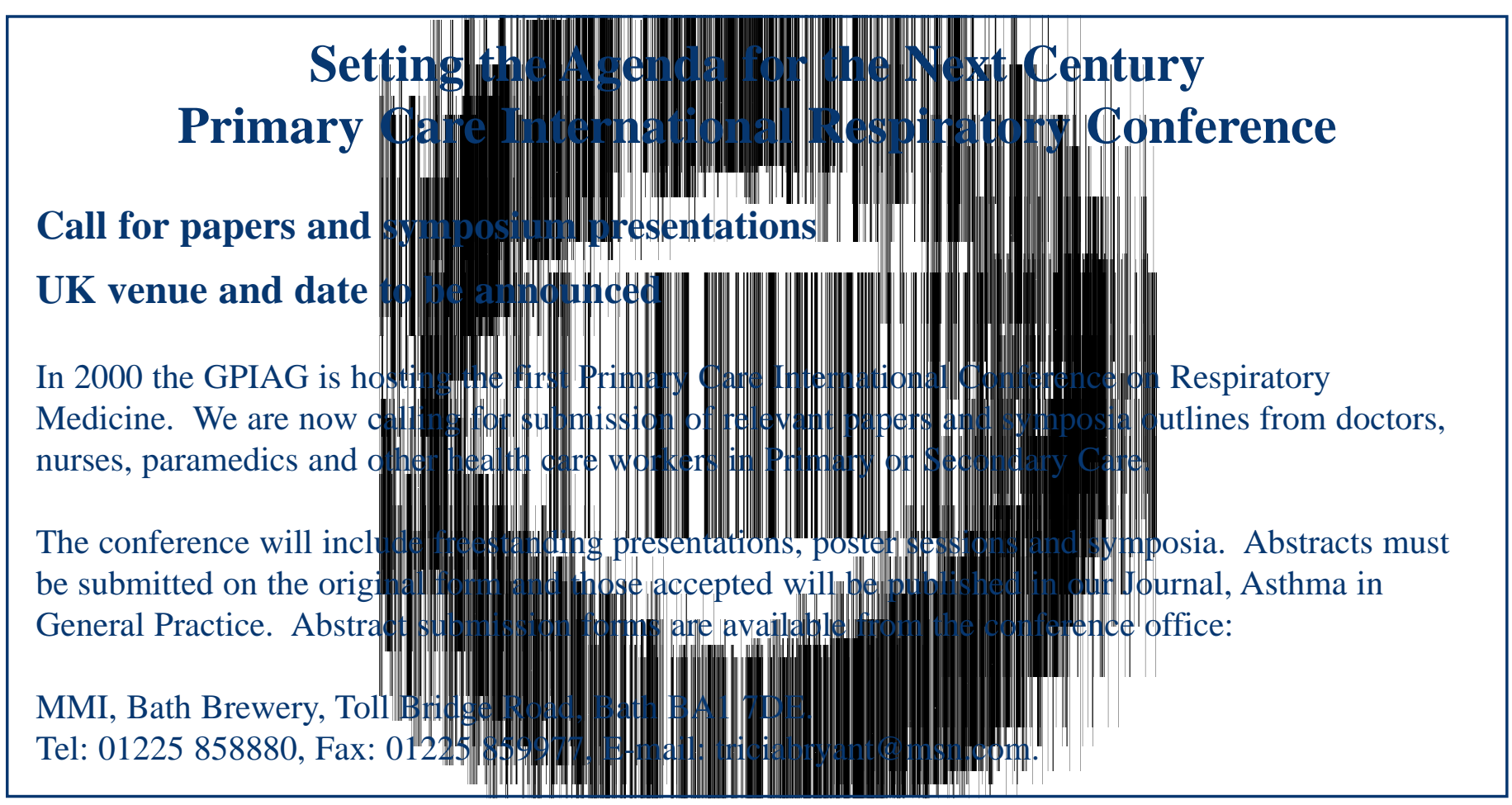

